\title{
The history of the Galactic bulge
}

\author{
A. A. Zijlstra ${ }^{1}$, K. Gesicki ${ }^{2}$ and M. M. Miller Bertolami ${ }^{3}$ \\ ${ }^{1}$ School of Physics \& Astronomy, University of Manchester, Oxford Road, Manchester \\ M13 9PL, UK \\ ${ }^{2}$ Centre for Astronomy, Faculty of Physics, Astronomy and Informatics, Nicolaus Copernicus \\ University, Grudziadzka 5, PL-87-100 Torun, Poland \\ ${ }^{3}$ Instituto de Astrofísica de La Plata, UNLP-CONICET, Paseo del Bosque s/n, 1900 La Plata, \\ Argentina
}

\begin{abstract}
Planetary nebulae form in stellar populations with ages from 1 to 10 Gyr, and can be used to trace their star formation histories. Here we apply this to the Galactic bulge, where there are indications both for an old origin and for younger stars. We use new stellar models, which have significant different evolutionary speeds during the post-AGB phase. We apply these new models to a sample of 32 planetary nebulae with HST imaging and VLT spectroscopy. The results show evidence for an old starburst, followed by continuous star formation until at least 2 Gyr ago. This agrees very well with recent analysis of colour-magnitude diagrams of the bulge. We show that the new models can also explain the [OIII] luminosity functions, and predict the uniform luminosity cut-off both in spiral galaxies and old elliptical galaxies.
\end{abstract}

Keywords. planetary nebulae: general, Galaxy: bulge, Galaxy: evolution

\section{Introduction}

The origin of the Galactic bulge remains a controversial issue. For a long time, the central bulge was considered an old component of the Milky Way galaxy. Several studies found that the colour-magnitude diagram are consistent with an old population, of approximately $10 \mathrm{Gyr}$. But there are also indications for a much younger population, for instance from spectroscopy of micro-lensed main-sequence stars. The controversy is reviewed in Nataf (2016).

Planetary nebulae $(\mathrm{PNe})$ form an important part of the puzzle of the bulge. There are currently $785 \mathrm{PNe}$ known in the bulge; the total $\mathrm{PNe}$ population is estimated at 2000-3500. PNe form the end-phase of low- and intermediate mass stars. These stars lose their envelopes on the AGB, and the remaining stellar cores quickly heat up and ionise the ejecta, before becoming white dwarfs. If the masses of the stellar cores can be determined, the initial-final mass relations can be used to derive initial masses. The formation ages of the stars come from the stellar life time vs stellar mass relation. From this the star formation history can be derived.

This was done by Gesicki et al. (2014), but it resulted in an unusual age of order 1 Gyr. For older stars, with lower stellar masses, the stellar models (Bloecker 1995) evolve too slowly from the AGB to the PN phase, so that the ejecta are dispersed before ionization begins. Gesicki et al. suggest that these models must underestimate the speed of evolution during the post-AGB phase, and make the PN stars appear too young. A comparison with a few asteroseismologically-determined masses suggest that the models are a factor of 3 too slow. This adjustment gives bulge ages between 3 and $10 \mathrm{Gyr}$.

Recently, new stellar evolution models for the post-AGB phase were published. With these models, we revisit the age of the Galactic bulge as determined from PNe. 

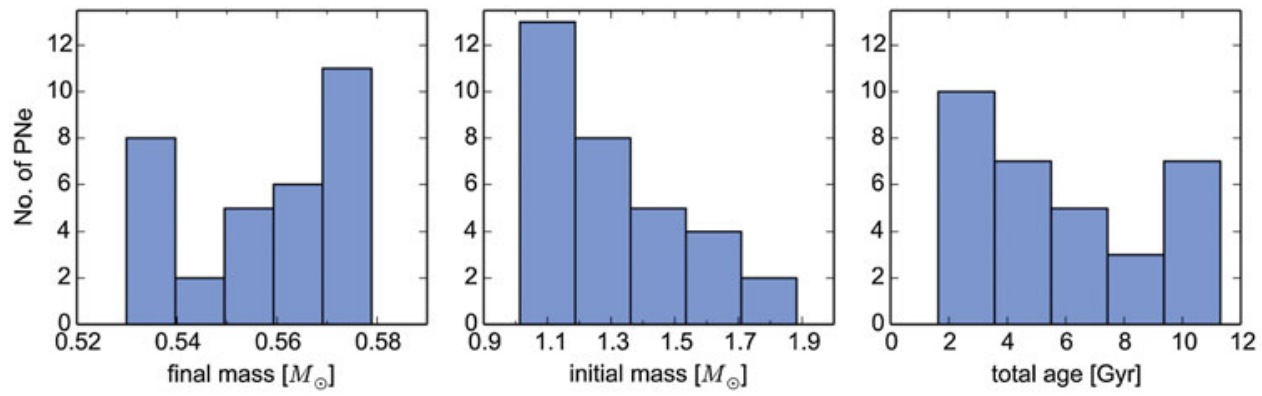

Figure 1. The core mass, initial mass, and stellar age distribution of the 32 Bulge PNe.

\section{Stellar models and methods}

The previous models for post-AGB evolution are based on the models of Schönberner (Blöcker 1995 and references therein) and Vassiliadis \& Wood (1994). A new series of models was recently published by Miller Bertolami (2016) using updated opacities. For the same core mass, the new models are brighter and cross the HR diagram much faster. The two aspects are related, as the evolutionary speed is set by the rate at which the envelope mass is digested, which is determined by the rate of nuclear burning and thus the luminosity. Compared to the old tracks, the post-AGB evolution is accelerated by a factor of 3 to 8 (larger at lower masses). This factor is close to that predicted by Gesicki et al. (2014). We use the set of new tracks, interpolating between them to obtain a denser grid in core mass.

The so-called Torun method is used in the analysis (Gesicki et al. 214). A photoionization model is used to reproduce the emission-line spectrum, line fluxes and line images of a PN. Good resolution images and a known distance significantly improve this modeling. The photo-ionization model returns the ionization structure, the density profile and the electron temperature distribution. Subsequently, the line profiles are used to fit a model for the internal velocity field. This requires echelle spectra of lines of different ionization stages. The velocity field, density distribution and outer radius are used to derive an expansion age. This includes a correction factor to account for the acceleration of the outer regions, as derived from hydrodynamical models (Schönberner 2016, 2005).

The photo-ionization model also returns the temperature of the star. The PN expansion age and current stellar temperature are compared to the post-AGB model tracks, to derive the best-fitting stellar core mass. The heating rate is a very strong function of stellar mass, making this method more accurate than plotting the PNe on the HR diagram (Gesicki \& Zijlstra 2007). The new stellar models have produced a systematic shift in the stellar mass vs heating rate relation.

Once the core masses are known, an initial-final-mass relation is used to obtain initial masses. Each initial mass corresponds to a different age of the star. The PN population yields the stellar death rates for that age. Combined with an initial mass function (a Salpeter function) this converts to a star formation rate for each age.

\section{Results}

We used a sample of 32 bulge PNe for which HST images and VLT/UVES echelle spectra are available. The original imaging program selected targets randomly from the Acker catalog, with the only constraints a position towards the Galactic bulge, and an angular diameter of less than 5 arcsec.

The final stellar masses, initial masses and age distribution of the sample are shown in Fig. 1. The stellar masses are significantly lower than found before, thanks to the 


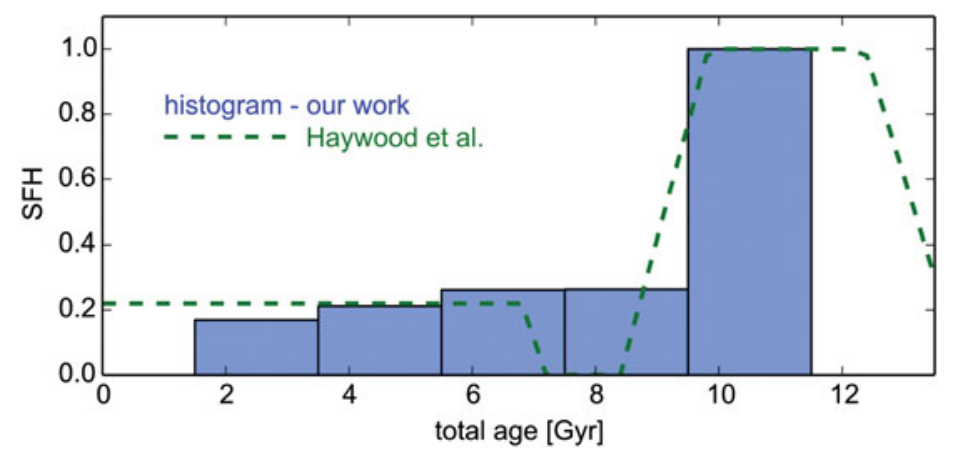

Figure 2. The normalized star formation history (SFH) of the Galactic bulge.

new stellar models. Stellar ages are between 2 and 12 Gyr, in better agreement with the expectations for the bulge than had previously been found. Still, the PN population cannot be solely due to an old population. A significant fraction is from younger stars.

To convert the age distribution to a star formation rate requires one final correction, regarding observational bias. This is the visibility time of the PN, described in detail in Gesicki et al. (2014): it takes into account that the time a PN remains visible is a function of stellar core mass, and is limited by how long it takes before the onset of ionization, and the duration of the phase before hydrogen burning ceases. The selection criterion on angular diameter also feeds into the visibility correction.

The resulting star formation history $(\mathrm{SFH})$ is shown in Fig. 2. We find that the SFH shows a peak at ages appropriate for the traditional 'old' bulge, but there has been continuous star formation at a lower rate since. We cannot say whether the star formation continued over the past 2 Gyr.

This SFH is indicative of a mixed bulge, partly formed in a short burst during the early phase of the Milky Way, and partly consisting of younger stars. The latter may be related to the disk population, scattered into the bulge by the action of bar: this is the main explanation for a 'pseudo' bulge, a thickened inner region of a disk.

Fig. 2 also shows the SFH proposed by Haywood et al. (2016), based on modeling of colour-magnitude diagrams. This is in excellent agreement with the results derived from PNe. The results are a good validation of the new tracks, and show that PNe can be used as tracers of old and intermediate-age populations.

\section{The O3 luminosity function}

The new models are also important for extragalactic PN populations. The PN luminosity function (PNLF) in other galaxies shows a sharp cut-off at an [OIII] magnitude around -4 : the cut-off magnitude is invariant across a range of galaxy types, from spirals to ellipticals (see also Mendez, these proceedings). With the older stellar models, this invariance was hard to understand. With the Blöcker tracks, the bright PNe come from young stars, and these are absent in elliptical galaxies (Ciardullo 2012). The new tracks can fit the PNLF with older stars.

Fig. 3 shows the PNLF as obtained from the new tracks. For each track, we have assumed a constant density, non evolving nebula, and calculated a photo-ionization model for a dense grid of stellar temperatures. For each model, we calculate the [OIII] flux. The method assumes that the nebula remains optically thick to ionizing photons and therefore will fail for the most evolved nebulae and possibly at the lowest masses where the nebulae are already evolved by the time ionization starts. 


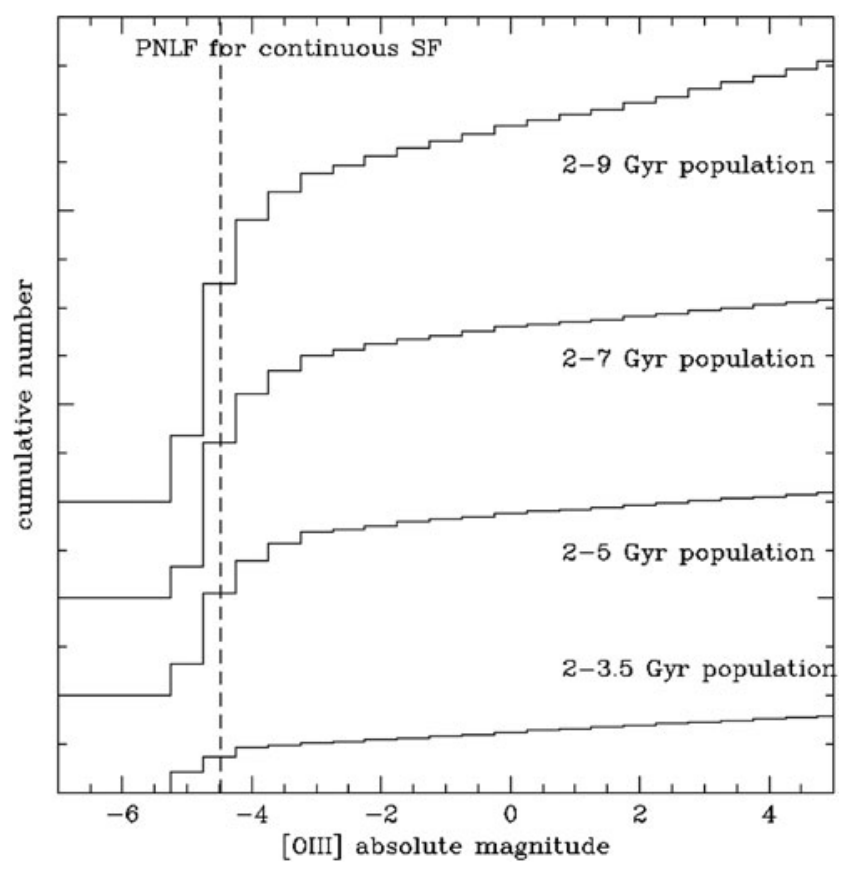

Figure 3. The [OIII] luminosity function of PNe, derived using the new post-AGB tracks.

The PNLF is shown for populations of a range of ages, assuming a constant star formation history. The dashed line shows the observed cut-off. The models reproduce this very well. The youngest stellar populations extend to brighter PNe, and populations older than 7 Gyr do not quite reach the cut-off luminosity.

For ages between 2 and 7 Gyr, the models confirm the presence of the cut-off at an invariant magnitude. This resolves a major problem in PN research. The new models show that the PNLF will give the same cut-off in all galaxy types, excluding those with exclusively stars older than $8 \mathrm{Gyr}$, and starburst galaxies which are dominated by very young stars. The results in this paper suggest the PNLF will also work for the Galactic bulge, based on the presence of a population younger than 8 Gyr.

\section{References}

Bloecker, T. 1995, A\&A, 299, 755

Ciardullo, R. 2012, ApSS, 341, 151

Gesicki, K. \& Zijlstra, A. A. 2007, A\&A, 467, L29

Gesicki, K., Zijlstra, A. A., Hajduk, M., \& Szyszka, C. 2014, A\&A, 566, A48

Haywood, M., Di Matteo, P., Snaith, O., \& Calamida, A. 2016, A\&A, 593, A82

Miller Bertolami, M. M. 2016, A\&A, 588, A25

Nataf, D. M. 2016, PASA, 33, e023

Schönberner, D., Jacob, R., \& Steffen, M. 2005, A\&A, 441, 573

Schönberner, D. 2016, Journal of Physics Conference Series, 728, 032001

Vassiliadis, E. \& Wood, P. R. 1994, ApJS, 92, 125

\section{Discussion}

ARNABOLDI: Are you predicting the morphology of the PNLF as the population ages? Are you also computing the deviations from the Ciardullo's function when the population is rejuvinated? 